\title{
Prenatal Detection of Congenital Fetal Heart Diseases using 2D greyscale ultrasound combined with Doppler in a high-risk population
}

\author{
Alshaimaa A. Albadawy, Esam I. Ali, Ameer A. Abdallah, \\ Moemen M. Hasan and Saad El Gelany \\ Department of Obstetrics and Gynecology, Faculty of Medicine, Minia University, Minia, Egypt
}

\begin{abstract}
Aim: We aimed to assess the accuracy and predictive value of fetal cardiac screening for congenital heart disease (CHD) at 18-22 gestational weeks in high-risk patients. Methods: In this study of fetal cardiac screening, four-chamber view, three-vessel view, outflow tracts followed by Doppler were recorded by obstetricians at 18-22 gestational weeks. A total of 438 fetuses that were scheduled for delivery at our hospital were included. Results: Forty-four newborns were born with CHD (10.9\%). The prenatal detection rate of CHD was (72.7\%). Although $80 \%$ of structural abnormality was diagnosed antenatally, only $44.4 \%$ of valvular abnormality was diagnosed. Conclusion: Detailed fetal heart scan performed by skilled obstetricians had a high detection rate for structural rather than valvular CHD in high-risk populations. The integration of this scan with multiple appropriate cardiac views into routine second trimester scan may improve the detection rate of fetal CHD and give a chance for parental counseling.
\end{abstract}

Keywords: congenital heart disease, four-chamber view, prenatal diagnosis, three-vessel view, outflow tract, Doppler

\section{Introduction}

CHD is considered a prominent cause of infant deaths. The incidence of CHD is likely about 4 13 per 1000 live births ${ }^{[1-3]}$. Between 1950 and 1994, $42 \%$ of infant deaths reported to the World Health Organization were attributable to cardiac defects ${ }^{[4]}$.

Prenatal Detection of CHD plays a substantial role in improving the outcome of fetuses with specific types of cardiac lesions [5-8]. Prenatal Detection of severe CHD enables a smooth transition of the patients from obstetricians to neonatologists or pediatric cardiac surgeons, leading to improved outcomes in patients with severe $\mathrm{CHD}^{[9,10] \text {. }}$

However, prenatal detection rates vary widely ${ }^{[11]}$. Some of this variation can be attributed to differences in examiner experience, maternal obesity, transducer frequency, abdominal scars, gestational age, amniotic fluid volume, and fetal lie ${ }^{[12,13]}$

The present study aimed to evaluate the CHD detection rate of the four-chamber, three-vessel views, and Doppler in a high-risk group during the second trimester.

\section{Patients and methods}

This study was a prospective cohort study of fetal cardiac screening. The study was carried out in The Feto- maternal unit of Obstetrics and Gynecology department, faculty of medicine, Minia University in Egypt. The type of ultrasound equipment used in fetal heart screening was standardized among all patients; Voluson S8 (GE Healthcare, Chicago, Illinois, USA). Besides, the type of ultrasound equipment used in postnatal echocardiography was standardized among all infants; Vivid T8 R2.5 cardiac ultrasound (GE Healthcare, American factories in China).

As projects must be authorized by the local research Ethics com-mittee (REC) in the department of Obstetrics \& Gynaecology before commence-ment, ethical permission was taken from the local ethical committee, and it approved the study protocol. The potential benefits and inconveniences of all aspects of the study were clearly stated in the patient

Prenatal Detection of Congenital Fetal Heart Diseases using 2D greyscale ultrasound combined with Doppler in a high-risk population 
information sheets, and each participant was provided written informed consent.

This project was submitted for review to the Ethics Committee under code (MUEOG0008). Sample size: was calculated using an online epitools program for "cohort studies sample size calculation" Using the following parameters; expected incidence in unexposed: 0.2 , Assumed relative risk: 1.5, Confidence interval: 0.95 , Desired power: 0.8. The probability of less than 0.05 was used as a cut off point for all significant tests. The minimal required sample size was (358). Our study included (402) cases. All patients attending the ANC clinic or FMU unit from January 2019 to January 2020 with at least one risk factor were included in the study.

A total of 438 pregnant participants were enrolled in this study during the period from January 2018 till January 2019. Patients with gestational age between 18-22 weeks having at least one risk factor from those listed in table (1) were recruited while visiting either the ANC clinic or the feto-maternal unit in Minia University Hospital (Fig.1)

Table 1.Risk factors for $\mathrm{CHD}^{[14]}$
All patients had a detailed 2D/greyscale imaging of all cardiovascular structures by skilled obstetric physician sonographers starting by the four-chamber view followed by both right and left outflow tracts ending by the threevessel view. Also, colour Doppler interrogation of all the valves, veins, arteries, atrial and ventricular septae was done. Pulsed Doppler of the valves and Ductus venosus was done. A detailed anomaly scan of fetal organs was performed to detect other associated extracardiac anomalies. After birth, all neonates were clinically examined by a neonatologist. A pulse oximeter was used to monitor the oxygen saturation levels in the neonates. A complete cardiac examination was done. Then neonates were subjected to echocardiography at one month's age. Thirty-six cases were excluded from the analysis( 14 cases in which newborn information was unavailable, 17 cases of termination of pregnancy, and five cases of intrauterine fetal death). Ultimately, 402 neonates who underwent fetal cardiac screening at 18-21 GW and heart examination at the 1month postnatal checkup were eligible for the study (Fig. 2).

\begin{tabular}{|l|l|}
\hline \multicolumn{2}{|l|}{} \\
\hline $\mathbf{1 .}$ & Pre-gestational Diabetes Miletus \\
\hline $\mathbf{2 .}$ & Gestational Diabetes Miletus \\
\hline $\mathbf{3 .}$ & LupuslSjogren's \\
\hline $\mathbf{4 .}$ & Hypothyroidism \\
\hline $\mathbf{5 .}$ & Teratogens \\
\hline $\mathbf{6 .}$ & Maternal infection \\
\hline $\mathbf{7 .}$ & Assisted Reproductive Technique \\
\hline $\mathbf{8 .}$ & Family history (Paternal or Maternal) of CHD \\
\hline $\mathbf{9 .}$ & Sibling with structural disease \\
\hline $\mathbf{1 0 .}$ & Rhythm or Rate abnormalities detected in U/S \\
\hline $\mathbf{1 1}$. & Noncardiac abnormality \\
\hline $\mathbf{1 2 .}$ & Abnormality of umbilical cord or placenta \\
\hline $\mathbf{1 3 .}$ & Monochorionic twins \\
\hline $\mathbf{1 4 .}$ & Hydrops Fetalis \\
\hline $\mathbf{1 5 .}$ & Known or suspected chromosomal abnormality \\
\hline $\mathbf{1 6 .}$ & Increased Nuchal Translucency \\
\hline
\end{tabular}




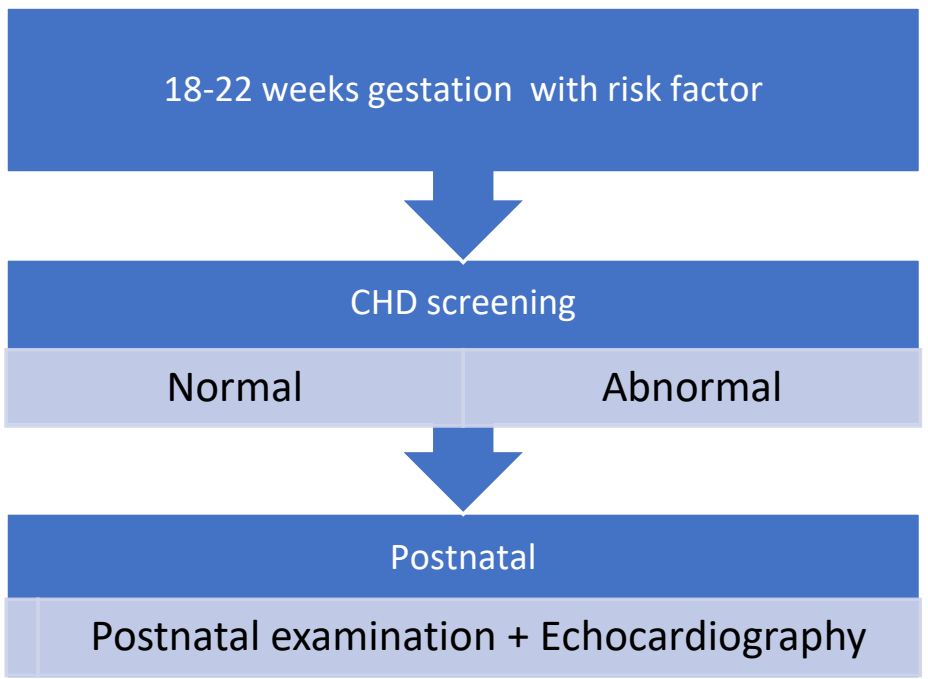

Fig (1) Flow chart of Fetal Heart screening. CHD: congenital heart disease

\section{8 patients were enrolled}

\section{8 patients underwent fetal heart screening}

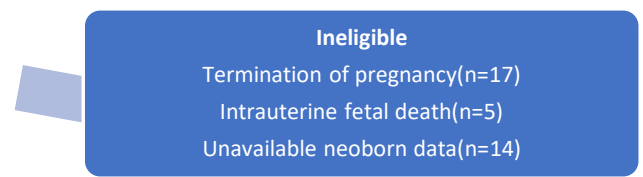

\section{2 eligible patients}

Fig. (2) Enrolment of study participants

The pregnancy outcome for all participants was obtained from the feto-maternal unit records. The incidence of CHD among high-risk patients was calculated. Besides, the prenatal detection rate of CHD was assessed. As a secondary outcome, we calculated the sensitivity, specificity, and predictive value of each of the fourchamber view, both right and left outflow tracts, three-vessel view and colour doppler in using 2D greyscale ultrasound combined with Doppler in a high-risk population 
patients was $29.09 \pm 5.7$. CHD was identified in 44 of 402 cases (10.9\%). The details of all CHD cases are listed in (Table 2). Thirty-six cases were diagnosed prenatally. The detection rate of antenatal diagnosis was (72.7\%). Although 28 of the 35 cases $(80 \%)$ of severe structural abnormalities were diagnosed prenatally, the prenatal detection rate of valvular abnormalities was $44.4 \%$ representing four of nine cases (Table 3). After excluding fetuses with structural abnormalities, we assessed the effectiveness of fetal cardiac screening based on the combination of the four-chamber view with the three-vessel view.

As several CHD, such as valvular abnormalities, might yield no findings in either of these views at 18-21 GW.1 Of the four cases that were prenatally detected, two showed abnormal findings in both the four-chamber and the three-vessel views, one showed abnormal RVOT, and another showed abnormal LVOT.

However, all prenatally diagnosed cases showed abnormal doppler. The specificity and positive predictive value of all views were $100 \%$. The sensitivity varied widely between different views. The diagnostic performance of all views is presented in (table 4, fig. 4). The prenatally diagnosed CHD types included 14 VSD, two ASD, three AVSD, two TGA, one PS, three TOF, two DORV, two HLHS, two TR, and one AS. Two ASD, one AVSD, one TAC, three TOF, two Ebstein anomaly, one TR, one AS and one PS was not prenatally diagnosed.

\section{Discussion}

This prospective cohort study of fetal cardiac screening where a total of 438 pregnant participants were enrolled; demonstrated that detailed fetal echocardiography performed by skilled obstetrician sonographers has high detection rates for CHDs in high-risk populations. The Feto-maternal unit of Obstetrics and Gynecology department, faculty of medicine, Minia University in Egypt has a situation where second-trimester ultrasound screening is performed by obstetricians trained in obstetric ultrasonography and working in a tertiary hospital rather than by technician sonographers. This study is considered a pilot study of our unit. 
Table 2. Summary and List of congenital heart diseases

\begin{tabular}{|c|c|c|c|c|c|c|c|}
\hline $\begin{array}{c}\text { Congenital heart disease } \\
\mathbf{N}(\%) \\
44(100 \%) \\
\end{array}$ & Category & $\begin{array}{c}\mathrm{CCV} \\
\text { Detection } \\
\text { Rate }\end{array}$ & $\begin{array}{l}\text { 3VV Detection } \\
\text { Rate }\end{array}$ & RVOT & LVOT & Doppler & $\begin{array}{c}\text { Prenatal } \\
\text { Detection } \\
\text { Rate }\end{array}$ \\
\hline $\begin{array}{c}\text { VSD } \\
14(31.8 \%)\end{array}$ & Structural & $100 \%$ & $0 \%$ & $0 \%$ & $0 \%$ & $100 \%$ & $100 \%$ \\
\hline $\begin{array}{c}\text { ASD } \\
4(9.1 \%) \\
\end{array}$ & Structural & $50 \%$ & $0 \%$ & $0 \%$ & $0 \%$ & $0 \%$ & $50 \%$ \\
\hline $\begin{array}{c}\text { AVSD } \\
4(9.1 \%)\end{array}$ & Structural & $75 \%$ & $0 \%$ & $0 \%$ & $0 \%$ & $75 \%$ & $75 \%$ \\
\hline $\begin{array}{c}\text { PS } \\
2(4.5 \%)\end{array}$ & Valvular & $50 \%$ & $50 \%$ & $50 \%$ & $0 \%$ & $50 \%$ & $50 \%$ \\
\hline $\begin{array}{c}\text { TAC } \\
1(2.3 \%)\end{array}$ & Structural & $0 \%$ & $0 \%$ & $0 \%$ & $0 \%$ & $0 \%$ & $0 \%$ \\
\hline $\begin{array}{c}\text { TGA } \\
2(4.5 \%)\end{array}$ & Structural & $0 \%$ & $0 \%$ & $100 \%$ & $100 \%$ & $100 \%$ & $100 \%$ \\
\hline $\begin{array}{c}\text { TOF } \\
6(13.6 \%)\end{array}$ & Structural & $16.7 \%$ & $50 \%$ & $50 \%$ & $50 \%$ & $50 \%$ & $50 \%$ \\
\hline $\begin{array}{c}\mathbf{T R} \\
3(6.8 \%)\end{array}$ & Valvular & $0 \%$ & $0 \%$ & $0 \%$ & $0 \%$ & $66.7 \%$ & $66.7 \%$ \\
\hline $\begin{array}{c}\text { AS } \\
2(4.5 \%)\end{array}$ & Valvular & $50 \%$ & $50 \%$ & $0 \%$ & $50 \%$ & $50 \%$ & $50 \%$ \\
\hline $\begin{array}{l}\text { DORV } \\
2(4.5 \%)\end{array}$ & Structural & $0 \%$ & $100 \%$ & $100 \%$ & $100 \%$ & $100 \%$ & $100 \%$ \\
\hline $\begin{array}{c}\text { HLHS } \\
2(4.5 \%) \\
\end{array}$ & Structural & $100 \%$ & $0 \%$ & $0 \%$ & $0 \%$ & $100 \%$ & $100 \%$ \\
\hline $\begin{array}{c}\text { Ebstein anomaly } \\
2(2.5 \%)\end{array}$ & Valvular & $0 \%$ & $0 \%$ & $0 \%$ & $0 \%$ & $0 \%$ & $0 \%$ \\
\hline
\end{tabular}

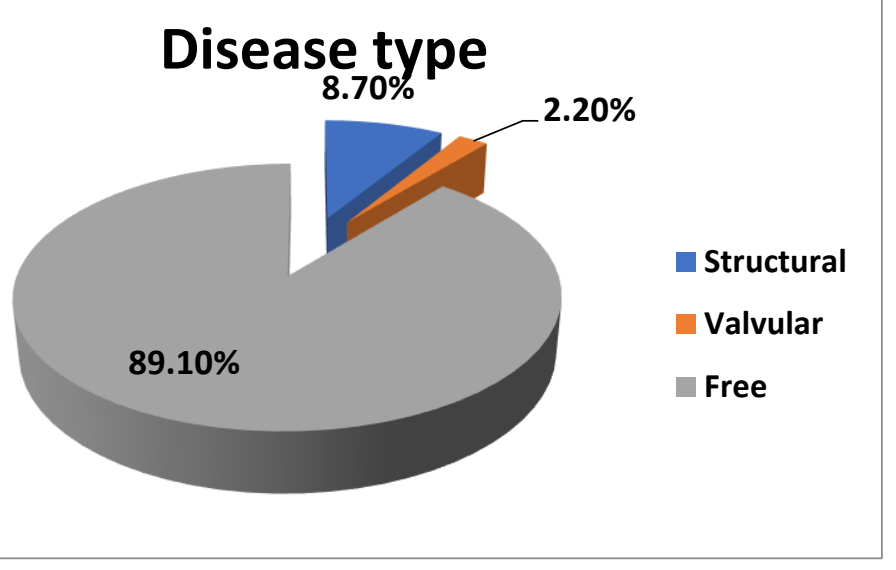

Fig. (3) Types of Congenital Heart Diseases 
MJMR, Vol. 31, No. 4, 2020, pages (59-67).

Table 3. Prenatal Diagnosis of both structural and valvular heart diseases

\begin{tabular}{|l|c|c|c|}
\hline & Structural & Valvular & Significance \\
\hline Total Number & $35(8.7 \%)$ & $9(2.2 \%)$ & $\begin{array}{l}\mathrm{P}=0.001^{*} \\
\chi 2=295.24\end{array}$ \\
\cline { 1 - 3 } $\begin{array}{l}\text { Prenatally } \\
\text { diagnosed }\end{array}$ & $28(80 \%)$ & $4(44.4 \%)$ & \\
\cline { 1 - 3 } Not diagnosed & $7(20 \%)$ & $5(55.6 \%)$ & \\
\hline
\end{tabular}

Table 4. Sensitivity and specificity of different views

\begin{tabular}{|l|c|c|c|c|c|}
\hline & Four CV & LVOT & RVOT & Three VV. & $\begin{array}{c}\text { All view } \\
\text { combined with } \\
\text { Doppler }\end{array}$ \\
\hline Sensitivity & $54.5 \%$ & $18.2 \%$ & $18.2 \%$ & $15.9 \%$ & $68.2 \%$ \\
\hline Specificity & $100 \%$ & $100 \%$ & $100 \%$ & $100 \%$ & $100 \%$ \\
\hline Positive predictive value & $100 \%$ & $100 \%$ & $100 \%$ & $100 \%$ & $100 \%$ \\
\hline Negative predictive value & $94.7 \%$ & $90.9 \%$ & $90.9 \%$ & $90.6 \%$ & $96.2 \%$ \\
\hline
\end{tabular}

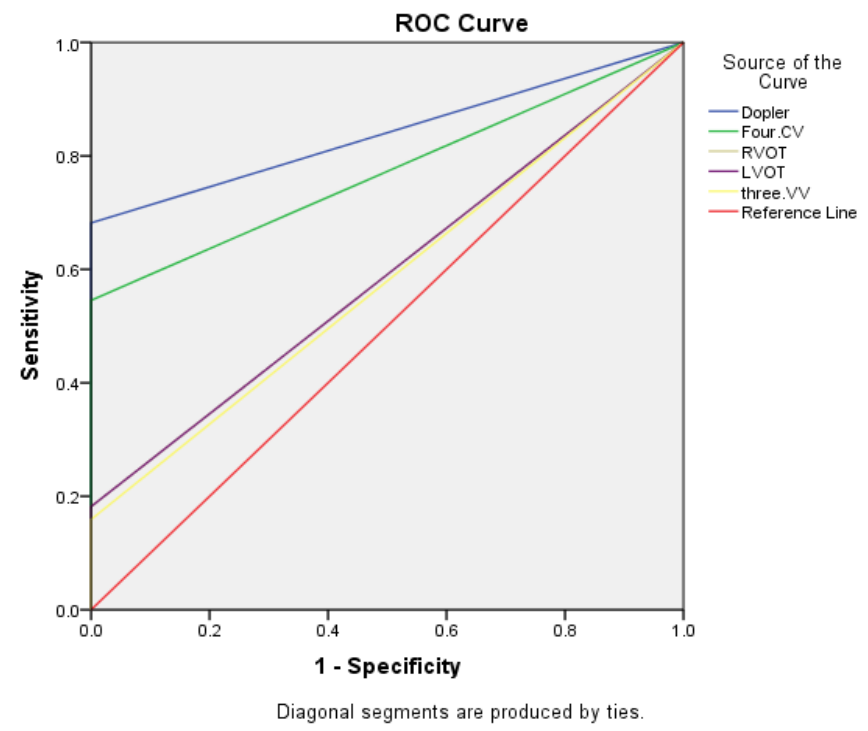

Fig 4. ROC Curve of Specificity and Sensitivity

In our study, a standardized protocol was used to perform fetal echocardiography, which included two-dimensional imaging, color flow mapping, and pulsed-wave Doppler. All 402 fetuses that were included in the data analysis had a final diagnosis of CHD confirmed or rebutted by postnatal echocardiography (regardless of whether the babies had clinical symptoms or cardiac murmurs or not). This approach leads to a more accurate evaluation of fetal echocardiography.
However, because of the socioeconomic circumstances, the feasibility and costeffectiveness of detailed fetal heart screening appear to be a matter of debate. The incorporation of multiple cardiac views into the routine ultrasound screening could be a good starting point.

By using a combination of the four-chamber view, three-vessel view, outflow tracts followed by Doppler, the prenatal detection rate of CHD was $(72.7 \%)$ for all cases. The prenatal detec-

Prenatal Detection of Congenital Fetal Heart Diseases using 2D greyscale ultrasound combined with Doppler in a high-risk population 
tion rate for structural abnormalities was $80 \%$, while for valvular abnormalities, it was $44.4 \%$. Itsukaichi, M., et al., ${ }^{[15]}$ recorded in their multicentric study a prenatal detection rate of $(75 \%)$ for structural abnormalities despite $(0 \%)$ for valvular abnormalities. However, They did not use Doppler in fetal heart screening.

The sensitivity for prenatal detection of CHD using a four-chamber view alone was $54.5 \%$, using the outflow tract view alone was $18.2 \%$, and when using a three-vessel view alone was 15.9\%. However, when adding Doppler to previous views, it became $68.2 \%$. The specificity for all views was $100 \%$ (Table 4).

Two decades ago, Stumpflen et al., ${ }^{[16]}$ in 1996 used routine detailed fetal echocardiography in 3085 unselected consecutive women and reported a sensitivity and specificity of 85.5 and $100 \%$, respectively, for the detection of any CHD.

Itsukaichi, M., et al., recorded that the sensitivity and specificity of the four-chamber view alone were $37.5 \%$ and $100 \%$, respectively, while those of the combination were $75.0 \%$ and $100 \%$, respectively.

Chu, C., et al., ${ }^{[17]}$ who used 2D multiple cardiac views and Doppler in 4264 high-risk patients and 5995 low-risk patients in 2017; documented that the overall sensitivity and specificity of fetal echocardiography in the prenatal detection of CHDs were 52.0 and $99.6 \%$, respectively, in the total study population, and 68.8 and $99.4 \%$, respectively, in the high-risk population.

Therefore, if detailed fetal echocardiography is incorporated into routine ultrasound screening and physician sonographers are trained with uniform standards, the prenatal diagnosis of CHD may be more effective in Minia. Our results are nearly similar to those of recent studies $^{[15,17]}$.

The accuracy of fetal cardiac screening in recent studies is higher than that in studies published over twenty years ago ${ }^{[18]}$. One reason for this improved accuracy is the dramatically enhanced performance of ultrasound devices ${ }^{[19]}$; especially, the resolution of ultrasound imaging has notably been upgraded. Another reason could be that numerous guidelines have been set up for sonographic screening examination of the fetal heart ${ }^{[14,20-22]}$

One of the limitations of our study is that despite recruiting and performing detailed fetal echocardiography in 438 pregnancies, complete follow-up data were only available for 402 fetuses/babies.

Also, for fetuses with known risk factors, the examiners may tend to be more careful and meticulous, which may also contribute to the improved sensitivity and specificity.

We were unable to detect seven cases of structural CHD (atrioventricular septal defect, tetralogy of Fallot, and truncus arteriosus communis) that would require perinatal management after birth. This may be because of differences in obstetricians' skills. Also, we were unable to detect five cases of valvular abnormalities, such as tricuspid regurgitation or pulmonary stenosis, aortic stenosis, and Ebstein anomaly.

It is known that some valvular abnormalities cannot be detected until at least 30 gestational weeks. Therefore, some fetal echocardiography guidelines, as in Japan, suggest that another fetal heart screening be performed at approximately $30 \mathrm{GW}^{[15]}$

The examiner's experience, quality of the ultrasound system, maternal body composition, fetal position, and the natural evolution of CHD may influence the detection rate. ${ }^{[17]}$

Nevertheless, the inclusion of the transverse and long axis of aortic arch views in fetal echocardiography may improve the detection rate of this CHD type to some extent. In the updated ISUOG practice guidelines in 2013, the three vessels and trachea view has been included in the sonographic screening examination of the fetal heart to enable the detection of aortic arch abnormalities ${ }^{[22]}$.

\section{Conclusion}

Single ultrasound screening of the fetal heart at 18-22 GW by obstetricians in the high-risk population could be beneficial for detecting severe structural rather than valvular abnormalities. However, this restriction may be

Prenatal Detection of Congenital Fetal Heart Diseases using 2D greyscale ultrasound combined with Doppler in a high-risk population 
resolved by conducting another fetal cardiac scan, including Doppler, at nearly $30 \mathrm{GW}$. The integration of fetal echocardiography with multiple appropriate cardiac views into routine second trimester scan may enhance the detection rate of fetal $\mathrm{CHD}$, provide parents with more information and allow for appropriate antenatal decision making.

\section{Disclosure}

The authors have stated clearly that there are no conflicts of interests in connection with this article.

\section{References}

1. Ferencz, C., et al., Congenital heart disease: prevalence at livebirth: the Baltimore-Washington Infant Study. American journal of epidemiology, 1985. 121(1): p. 31-36.

2. Meberg, A., et al., Outcome of congenital heart defects-a population-based study. Acta Paediatrica,2000.89(11):p.1344-1351.

3. Cuneo, B.F., et al., Trends in prenatal diagnosis of critical cardiac defects in an integrated obstetric and pediatric cardiac imaging center. Journal of perinatology, 2004. 24(11): p. 674-678.

4. Rosano, A., et al., Infant mortality and congenital anomalies from 1950 to 1994: an international perspective. Journal of Epidemiology \& Community Health, 2000. 54(9): p. 660-666.

5. Bonnet, D., et al., Detection of transposition of the great arteries in fetuses reduces neonatal morbidity and mortality. Circulation, 1999. 99(7): p. 916-918.

6. Andrews, R., et al., Outcome of staged reconstructive surgery for hypoplastic left heart syndrome following antenatal diagnosis. Archives of disease in childhood, 2001. 85(6): p. 474-477.

7. Franklin, O., et al., Prenatal diagnosis of coarctation of the aorta improves survival and reduces morbidity. Heart, 2002. 87(1): p. 67-69.

8. Tworetzky, W., et al., Balloon dilation of severe aortic stenosis in the fetus: potential for prevention of hypoplastic left heart syndrome: candidate selection, technique, and results of successful intervention. Circulation, 2004. 110(15): p. 2125-2131.
9. Tworetzky, W., et al., Improved surgical outcome after fetal diagnosis of hypoplastic left heart syndrome. Circulation, 2001. 103(9): p. 1269-1273.

10. Escobar-Diaz, M.C., et al., Prenatal diagnosis of transposition of the great arteries over a 20-year period: improved but imperfect. Ultrasound in Obstetrics \& Gynecology, 2015. 45(6): p. 678-682.

11. Simpson, L.L., Screening for congenital heart disease. Obstet Gynecol Clin North Am, 2004. 31(1): p. 51-9.

12. DeVore, GR, et al., Fetal echocardiography: factors that influence imaging of the fetal heart during the second trimester of pregnancy. Journal of ultrasound in medicine, 1993. 12(11): p. 659-663.

13. Sharland, GK and LD Allan, Screening for congenital heart disease prenatally. Results of a 2 $\frac{1}{2}$-year study in the South East Thames Region. BJOG: An International Journal of Obstetrics \& Gynaecology, 1992. 99(3): p. 220-225.

14. Donofrio, M.T., et al., Diagnosis and treatment of fetal cardiac disease: a scientific statement from the American Heart Association. Circulation, 2014. 129(21): p. 2183-2242.

15. Itsukaichi, M., et al., Effectiveness of fetal cardiac screening for congenital heart disease using a combination of the fourchamber view and three-vessel view during the second trimester scan. 2018. 44(1): p. 49-53.

16. Stümpflen, I., et al., Effect of detailed fetal echocardiography as part of routine prenatal ultrasonographic screening on detection of congenital heart disease. 1996. 348(9031): p. 854-857.

17. Chu, C., et al., Prenatal diagnosis of congenital heart diseases by fetal echocardiography in second trimester: a Chinese multicenter study. 2017. 96(4): p. 454-463.

18. Buskens, E., et al., Efficacy of routine fetal ultrasound screening for congenital heart disease in normal pregnancy. 1996. 94(1): p.67-72.

19. Rubesova, E. and R.A.J.A.j.o.p. Barth, Advances in fetal imaging. 2014. 31(07): p. 567-576.

20. Satomi, GJPI, Guidelines for fetal echocardiography. 2015. 57(1): p. 1-21. 
21. Sklansky, M.J.J.o.u.i.m.o.j.o.t.A.I.o.U.i.M., Current guidelines for fetal echocardiography: time to raise the bar. 2011. 30(2): p. 284-6; author reply 286 .
22. Carvalho, J., et al., ISUOG Practice Guidelines (updated): sonographic screening examination of the fetal heart. 2013. 